\title{
Coumadin alone or aspirin plus coumadin reduced coronary events and death after acute myocardial infarction or unstable angina
}

Sources of funding: The Netherlands National Health Insurance Fund Council and the Netherlands Heart Foundation.

For correspondence: Professor D Grobbee, University Medical Centre Utrecht, Utrecht, The Netherlands. d.e.grobbee@jc.azu.nl

Abstract and commentary also published in ACP Journal Club van Es RF, Jonker JJ, Verheugt FW, et al, for the Antithrombotics in the Secondary Prevention of Events in Coronary Thrombosis-2 (ASPECT-2) Research Group. Aspirin and coumadin after acute coronary syndromes (the ASPECT-2 study): a randomised controlled trial. Lancet. 2002;360:109-13.

QUESTION: In patients who have had acute myocardial infarction (MI) or unstable angina, is high intensity coumadin or aspirin plus moderate intensity coumadin more effective than aspirin alone for reducing coronary events and all cause mortality?

\section{Design}

Randomised (allocation concealed*), unblinded,* controlled trial with follow up to $\leq 26$ months.

\section{Setting}

53 hospitals in the Netherlands.

\section{COMMENTARY}

Clinical interest in coumadin therapy after MI declined in the 1990s after several studies showed no benefit over aspirin. Therefore, recent guidelines recommend the use of aspirin and other antiplatelet therapies (eg, clopidogrel) at discharge for patients with acute coronary syndromes (ACSs).

The ASPECT-2 study by van Es $e$ a $a$ and 2 other recent trials ${ }^{1-2}$ suggest that long term coumadin therapy compared with aspirin improves outcomes in patients after ACS. In ASPECT-2, fewer patients in both the aspirin plus coumadin group (hazard ratio 0.50, 95\% CI 0.27 to 0.92 ) and the coumadin alone group (hazard ratio 0.55 , CI 0.30 to 1.00 ) reached the primary end point (death, MI, or stroke) than those in the aspirin group after 12 months. The benefits from coumadin therapy were further supported by secondary analyses showing lower rates of death, vascular death, MI, unstable angina, stroke, and revascularisation in patients receiving coumadin or aspirin plus coumadin therapy than in those receiving aspirin alone. However, the aspirin plus coumadin group had a 2 fold higher rate of major bleeding, and a 3 fold higher rate of minor bleeding than the aspirin alone group.

Clinical application of the ASPECT-2 findings and widespread use of coumadin therapy after ACS are doubtful in contemporary cardiovascular practice. In ASPECT-2, bleeding concerns, study design, and eligibility criteria largely excluded patients receiving early revascularisation, intracoronary stents, or clopidogrel. Prohibition of such patients limits the determination of the additive benefit or therapeutic role of coumadin in ACS management. In addition, the narrow pharmacological efficacy of coumadin (ie, safety margin and requirement for prothrombin time/INR monitoring) reduces physician and patient acceptance. Consequently, ASPECT-2 is unlikely to propel coumadin to the forefront of ACS therapies ahead of aspirin and clopidogrel. The study should, however, stimulate development of new oral anticoagulants and additional comparison trials of antiplatelet-anticoagulant drug regimens.

Michael P Hudson, MD, MHS Henry Ford Heart Eं Vascular Institute Detroit, Michigan, USA

1 Hurlen M, Abdelnoor M, Smith P, et al. Warfarin, aspirin, or both after myocardial infarction. $N$ Engl J Med 2002;347:969-74

Brouwer MA, van den Bergh PJ, Aengevaeren WR, et al. Aspirin plus coumarin versus aspirin alone in the prevention of reocclusion after fibrinolysis for acute myocardial infarction: results of he Antithrombotics in the Prevention of Reocclusion In Coronary Thrombolysis (APRICOT)-2 Trial. Circulation 2002;106:659-65.

\section{Patients}

999 patients who had had acute MI (88\%) or unstable angina (13\%) within the preceding 8 weeks. Exclusion criteria included planned revascularization or recent intracoronary stenting, thrombocytopenia, anaemia, and history of stroke. Follow up was 99\% (mean age 61 y, $77 \%$ men)

\section{Intervention}

Patients were allocated to phenprocoumon or acenocoumarol with target international normalized ratio (INR) of 3.0 to 4.0 (coumadin group, $n=330$ ); aspirin, 80 $\mathrm{mg} /$ day, plus phenprocoumon or acenocoumarol with target INR of 2.0 to 2.5 (combination group, $\mathrm{n}=333$ ); or aspirin, $80 \mathrm{mg} /$ day (aspirin group, $\mathrm{n}=366$ ).

\section{Main outcome measures}

The primary outcome was a composite of all cause mortality, MI, or stroke. The major secondary outcome was bleeding.

\section{Main results}

Analysis was by intention to treat. The incidence of the composite outcome of all cause mortality, MI, or stroke was lower in the coumadin and combination groups than in the aspirin group (table). The incidence of minor bleeding was greater in the combination group than in the aspirin group (table). The groups did not differ for major bleeding (table).

\section{Conclusion}

In patients who have had acute myocardial infarction or unstable angina, high intensity coumadin alone or aspirin plus moderate-intensity coumadin was more effective than aspirin alone for reducing coronary events and all cause mortality.

*See glossary.

Coumadin or coumadin plus aspirin (combination group) $v$ aspirin alone in acute myocardial infarction or unstable angina at median follow up of 12 monthst

\begin{tabular}{|c|c|c|c|c|}
\hline Outcomes & Comparisons & Event rates & RRR $(95 \%$ Cl) & NNT (CI) \\
\hline \multirow[t]{2}{*}{ Composite end point } & Coumadin $v$ aspirin & $5 \%$ v $9 \%$ & $43 \%$ (1 to 68$)$ & 26 (13 to 2740$)$ \\
\hline & Combination $v$ aspirin & $5 \% \vee 9 \%$ & $48 \%$ (7 to 71$)$ & 23 (12 to 184$)$ \\
\hline \multirow[t]{2}{*}{ Major bleeding } & Coumadin $v$ aspirin & $1 \% \vee 1 \%$ & $3 \%(-76$ to 345$)$ & Not significant \\
\hline & Combination $v$ aspirin & $2 \% \vee 1 \%$ & $136 \%(-32$ to 733$)$ & Not significant \\
\hline \multirow[t]{2}{*}{ Minor bleeding } & Coumadin $v$ aspirin & $8 \% \vee 5 \%$ & $68 \%(-7$ to 205$)$ & Not significant \\
\hline & Combination $v$ aspirin & $15 \%$ v $5 \%$ & $216 \%$ (86 to 442$)$ & $10(7$ to 17$)$ \\
\hline
\end{tabular}

tComposite end point = all cause mortality, myocardial infarction, or stroke. Abbreviations defined in glossary; RRI, RRR, NNT, NNH, and Cl calculated from data in article. 